\title{
Réflexion multidisciplinaire sur la prise en charge du Déficit androgénique lié à l'âge
}

\author{
Jacques BUVAT, Pierre COSTA, Thierry FLAM, François GIULIANO, Jean-Marc KUHN, Erick \\ LEGRAND, Hervé LEJEUNE, Sylvain MIMOUN, Marc ROGER, Simon WEBER.
}

\begin{abstract}
Groupe multidisciplinaire : Dr Jacques BUVAT, endocrinologue, Lille, Pr Pierre COSTA, urologue, Nîmes, Pr Thierry FLAM, urologue, Paris, Dr François GIULIANO, urologue, Paris, Pr Jean-Marc KUHN, endocrinologue, Rouen,

Dr Erick LEGRAND, rhumatologue, Angers, Pr Hervé LEJEUNE, endocrinologue, Lyon, Dr Sylvain MIMOUN, andrologue, Paris, Dr Marc ROGER, biologiste, Paris, Pr Simon WEBER, cardiologue, Paris
\end{abstract}

\section{RÉSUMÉ}

La prise en charge du déficit androgénique lié à l'âge (DALA) comporte tout d'abord l'établissement précis du diagnostic. Celui-ci est évoqué devant des signes cliniques relativement peu spécifiques qu'il convient toutefois de reconnaître. Le diagnostic de DALA nécessite une confirmation biologique par des méthodes tenant compte des variations de la SHBG comme le dosage de la testostérone biodisponible ou, au moins, l'index de testostérone libre (testostérone totale/SHBG) ou encore, la testostérone libre calculée. Bien que la valeur seuil permettant de retenir le diagnostic de DALA n'ait pas été étudiée de manière spécifique, la valeur inférieure de la normale des hommes jeunes, qui est la valeur utilisée pour inclure les sujets dans les essais thérapeutiques, semble une valeur appropriée pour définir un déficit androgénique. Les données actuellement disponibles sont en faveur d'un avantage du traitement substitutif sur la qualité de vie, la sexualité, l'état métabolique, la composition corporelle et l'ostéoporose. La mise en place d'un traitement androgénique substitutif nécessite toutefois un dépistage soigneux du cancer de la prostate. La surveillance du traitement androgénique portera en particulier sur l'état prostatique et l'hématocrite et devra assurer une substitution physiologique. La prise en charge du patient devra aussi tenir compte du contexte pathologique susceptible d'intervenir comme facteur aggravant du DALA et de nécessiter une thérapeutique spécifique.

Mots clés : andropause, déficit androgénique, âge, testostérone

\section{INTRODUCTION}

Ce texte représente la conclusion de 18 mois de réflexions (juin 2001 - décembre 2002) d'un groupe multidisciplinaire d'experts français sur le thème encore très controversé il n'y a pas si longtemps du Déficit Androgénique Lié à l'Âge (DALA) dans ses différents aspects cliniques, diagnostiques et thérapeutiques. Cette réflexion a conduit à la rédaction d'une série de revues générales consacrées à chacun de ces aspects, qui ont été pour la plupart publiées en 2002 dans la revue Andrologie [4, 6, 10, 11, $13,15,19]$. Ce texte constitue une synthèse de ces revues où l'on trouvera une bibliographie récente extensive. Seules quelques références majeures seront reprises ici. Il est important de noter que pendant le déroulement de ce travail de réflexion sont parues plusieurs publications consacrées au DALA, basées soit sur des réunions de consensus [16] soit sur d'autres revues générales extensives [14], et qui rejoignent nos conclusions.

\section{PRÉSENTATION CLINIQUE DU DÉFICIT ANDROGÉNIQUE LIÉ À L'ÂGE}

La diminution de plusieurs hormones a été mise en évidence chez les sujets vieillissants. Ces déficits hormonaux sont volontiers dénommés en utilisant le suffixe « pause ». Ménopause et andropause correspondent ainsi à l'altération de la «fonction de reproduction » ce qui, au

Correspondance :

Pr. Hervé LEJEUNE - Département de Médecine de la Reproduction, Faculté de Médecine, Domaine Rockefeller, 8 avenue Rockefeller 69008 LYON - Tel 04.78.77.70.65 - Fax 04.78.77.72.64 Emaillejeune@univ-lyonl.fr 
sens large, englobe la fertilité, la sexualité et les fonctions modulées par les stéroïdes sexuels. Toutefois, l'altération de la fonction de reproduction est différente chez l'homme et chez la femme : alors que la ménopause se caractérise par une disparition obligatoire et définitive de la fonction de reproduction avec arrêt pratiquement complet de la sécrétion ovarienne de progestérone et d'estrogènes, « l'andropause » correspond à une diminution inconstante et, lorsqu'elle existe, partielle, progressive dans le temps et variable d'un homme à l'autre, de la sécrétion de testostérone avec conservation d'une possibilité de reproduction jusqu'à un âge avancé. Le terme d' " andropause » paraît donc peu adapté. Pour couper court à la controverse sémantique, les spécialistes lui préfèrent désormais un vocable plus descriptif de l'involution de la fonction gonadique masculine : hypogonadisme partiel de l'homme âgé ou Déficit Androgénique Lié à l'Âge (DALA) (pour références voir [13]). Dans la littérature anglo-saxonne, on rencontre également souvent la dénomination PADAM pour Partial Androgen Deficiency of the Aging Male.

Les conséquences cliniques du DALA sont variées, directement liées au rôle physiologique de la testostérone. La symptomatologie est toutefois d'installation insidieuse, d'évolution lente et d'intensité modérée en raison du caractère partiel du déficit androgénique. Ainsi les manifestations cliniques du DALA sont souvent difficiles à distinguer des autres manifestations du vieillissement [8]. Elles sont en outre fortement dépendantes du contexte psychosocial dans lequel évolue l'homme vieillissant, en particulier dans le domaine de la sexualité.

Pourtant un DALA emprunte à l'hypogonadisme franc un certain nombre de symptômes (Tableau 1), qui, sans être spécifiques, constituent néanmoins autant de signes d'appel $[12,17]$.

- des symptômes fonctionnels tels qu'asthénie et fatigabilité accrue, troubles du sommeil mais aussi accès d'hypersudation ou bouffées vasomotrices,

- une modification de la composition corporelle avec diminution de la masse maigre, de la masse et de la force musculaires, inflation de la graisse viscérale (surtout abdominale) (pour références voir [10]),

- une altération des caractères sexuels secondaires avec dépilation pubienne et axillaire, gynécomastie et hypotrophie testiculaire,

- des troubles sexuels représentés essentiellement par une diminution de la libido et de l'activité sexuelle, une altération de la qualité des érections en particulier une raréfaction ou une disparition des érections nocturnes et matinales spontanées, une diminution de l'intensité de l'orgasme et du volume de l'éjaculât,

- des troubles neuro-psychiques, le plus souvent sur un mode anxio-dépressif : irritabilité ou indifférence, perte
Tableau 1 : Manifestations cliniques du déficit androgénique lié à l'âge.

- Troubles sexuels avec une baisse de la libido et des fantasmes, une diminution/disparition des érections nocturnes et matinales au réveil, une altération de l'éjaculation (petit volume, retard au déclenchement, diminution de la sensation de plaisir) et une diminution de la qualité des érections.

- Modifications de l'apparence corporelle avec prise de poids (augmentation de la graisse abdominale et viscérale), diminution de la musculature, réduction de la pilosité corporelle et apparition d'une gynécomastie.

- Déminéralisation osseuse, diminution de la masse et de la force musculaire ainsi que de l'endurance, anémie, le tout pouvant limiter les activités physiques et sportives.

- Troubles neuro-végétatifs et de l'humeur avec asthénie, tendance dépressive, somnolence diurne, insomnie, nervosité.

de l'estime de soi, manque de motivation, d'élan vital et de combativité, troubles de la concentration, parfois, il s'agit de troubles de la mémoire récente (pour références voir [15]),

- l'accélération de la perte osseuse et le développement de l'ostéoporose exposent à un risque fracturaire accru (pour références voir [11]).

La plupart de ces symptômes peuvent s'expliquer par une diminution de la testostérone mais ne sont pas réellement pathognomoniques. Il est important de souligner que la chronologie de leur survenue est variable d'un homme à un autre. Ces signes sont d'autant plus importants à rechercher que le contexte est favorable à une baisse de la testostéronémie : alcoolisme, traitement corticoïde au long cours, maladies chroniques ...[22].

\section{UN TABLEAU CLINIQUE HÉTÉROGENE PEUT-IL GUIDER UN SCREENING BIOLOGIQUE ?}

Le tableau clinique qui vient d'être présenté apparaît très hétérogène ; surtout, il peut être lié au vieillissement sans qu'il y ait pour autant déficit en testostérone : à cet égard, on signalera que les troubles de l'érection qui sont plus fréquents avec l'âge, ne se trouvent associés à un hypogonadisme que dans $8,3 \%$ des cas en moyenne sur les grandes séries publiées [9]. Dans le même ordre d'idée, les troubles du sommeil et/ou les troubles anxio-dépressifs peuvent parfaitement se développer en dehors de tout contexte hormonal.

L'association de plusieurs de ces signes et symptômes motivant une plainte du patient sur sa qualité de vie justifie, chez un homme vieillissant, la recherche d'une baisse de la testostéronémie. 


\section{QUELS DOSAGES HORMONAUX DEMANDER ?}

Devant ce tableau symptomatique malaisé à objectiver et à quantifier sur le plan clinique, le diagnostic de DALA doit être confirmé par la mise en évidence de perturbations hormonales caractérisées.

Quels sont les paramètres à doser et quels dosages sont accessibles et fiables (pour références voir [19]) ?

La testostérone totale est égale à la somme de la testostérone liée à la SHBG (ou TeBG), de la testostérone liée à l'albumine et de la testostérone libre. La testostérone biodisponible correspond à la fraction liée à l'albumine et à la fraction libre. Chez l'homme jeune, les fractions liée à la SHBG et liée à l'albumine sont à peu près équivalentes (50/50). Chez l'homme âgé autour de 70 ans, en moyenne $60 \%$ de la testostérone est lié à la SHBG, $39 \%$ à l'albumine, et $1 \%$ est libre.

Le dosage de la testostérone totale est le plus classique et le plus facile à réaliser ; il reste le plus accessible car il existe de nombreuses méthodes non isotopiques sur automates. Chez l'homme adulte, il est considéré comme normal par la plupart des auteurs pour des valeurs comprises entre 3 et $10 \mathrm{ng} / \mathrm{ml}$. Dans une cohorte d'hommes de 20 à 49 ans à spermogramme normal et gonadotrophines normales, les $5^{\circ}$ et $95^{\circ}$ percentiles sont 3,4 et $10 \mathrm{ng} / \mathrm{ml}$ respectivement. La baisse des taux moyens s'observe déjà entre 30 et 40 ans et se poursuit très régulièrement. La testostérone totale diminue, en moyenne, de 1 à $2 \%$ par an.

Cependant, plusieurs données relativisent la valeur diagnostique de ce paramètre : la grande variabilité de la baisse des taux avec l'âge, la disparition du rythme circadien chez le sujet âgé, l'augmentation des taux circulants de SHBG et consécutivement de la testostérone liée à la SHBG, ce qui masque en partie la baisse du taux de testostérone biodisponible.

De plus, certaines situations font de ce dosage un reflet imparfait de l'imprégnation hormonale réelle de l'homme vieillissant : atteinte hépatique, obésité, interactions médicamenteuses... Il s'agit de situations dans lesquelles la SHBG est modifiée. Le dosage de la SHBG peut alors aider à l'interprétation de la testostérone totale. Le rapport Testostérone totale/SHBG appelé index de testostérone libre ou des formules calculées en tenant compte des affinités et capacités de liaison des protéines de liaison des stéroïdes (comme celle utilisable sur le site internet de l'International Society for the Study of the Aging Male : http://www.issam.ch/freetesto.htm) peuvent être utilisés. On remarque toutefois que ces méthodes combinent les imprécisions des deux dosages et posent le problème des normes qui sont souvent données pour chacun des dosages mais pas pour leur rapport ou pour les valeurs calculées.
La testostérone biodisponible (non liée à la SHBG), est le reflet le plus fiable de l'hypogonadisme lié à l'âge pour beaucoup d'auteurs. Sa valeur reflète l'imprégnation de l'organisme en testostérone en s'affranchissant des variations de la SHBG, mais son dosage, plus complexe, n'est réalisable que par des laboratoires expérimentés (précipitation plus RIA).

La testostérone libre, non liée à la SHBG ou à d'autres protéines plasmatiques comme l'albumine, serait le paramètre reflétant le mieux l'androgénicité au niveau des récepteurs et son éventuelle diminution liée à l'âge. La méthode de dosage la plus fiable (dialyse à l'équilibre plus RIA) est hors de portée de la plupart des laboratoires, compte tenu de sa difficulté de mise en cuvre. Les méthodes plus simples dites avec analogue, utilisées par certains laboratoires, se sont avérées en fait non fiables (pour références voir [19]).

Le dosage de la LH peut venir en aide au diagnostic d'hypogonadisme lié à l'âge.

En cas d'hypogonadisme, une LH élevée affirme un hypogonadisme par atteinte primitive du testicule et une LH normale ou basse, un hypogonadisme par insuffisance gonadotrope, ces deux types de déficit pouvant être observés chez le sujet âgé.

En cas de testostérone située à la limite inférieure de la norme, un taux élevé de $\mathrm{LH}$ confirme la réalité d'une défaillance testiculaire partiellement compensée par l'augmentation de cette hormone.

Le dosage de $\mathrm{LH}$ est inutile en première intention mais est justifié en association au second dosage de testostérone, demandé pour vérifier la constance des anomalies.

Les significations exactes et/ou les valeurs diagnostiques des autres paramètres sont trop discutées pour que leurs déterminations fassent l'objet de recommandations consensuelles :

Cela vaut pour la FSH et l'inhibine B. Même si un dosage spécifique de cette dernière montre un déclin de sa sécrétion au moins aussi précoce que celui de la testostérone et si les taux de FSH s'élèvent très progressivement au-delà de 50 ans, il ne s'agit là que d'un reflet d'un possible déficit de la spermatogenèse lié à l'âge, sans corrélation obligatoire avec le DALA. Au total, le dosage de FSH est peu ou pas contributif au diagnostic de l'hypogonadisme partiel de l'homme âgé.

Le dosage de l'androstanediol et de la DHEA n'a pas démontré d'intérêt en pratique clinique pour le diagnostic d'hypogonadisme.

La baisse de l'estradiol biodisponible apparaît comme un paramètre sensible du vieillissement endocrinien mais les méthodes de dosages ne sont pas réalisables en pratique de routine. 
Le dosage de la prolactine doit rester une règle en cas de baisse franche de la testostérone avec LH normale ou basse, car un hypogonadisme peut révéler une hyperprolactinémie, elle-même souvent conséquence d'un adénome hypophysaire ou d'une autre une pathologie organique de la région hypothalamo-hypophysaire nécessitant un bilan endocrinien et morphologique (IRM).

\section{QUAND UN TRAITEMENT ANDROGE- NIQUE SUBSTITUTIF PAR LA TESTOSTÉ- RONE DOIT-IL OU PEUT-IL ÊTRE INSTAURÉ ?}

La définition biologique du DALA est une question clé car d'elle dépendra l'attitude thérapeutique. La référence doitelle être la norme de la testostéronémie de l'adulte jeune ou, au contraire, la fourchette des valeurs normales rapportées à l'âge ? Quel critère analyser ?

La valeur seuil permettant de retenir le diagnostic de déficit androgénique n'est pas facile à déterminer.

Les travaux mettant en relation les valeurs de testostéronémie et les signes cliniques montrent que ces relations suivent des modèles différents selon les effets étudiés [3].

Une relation dose-effet est observée de manière linéaire pour les caractéristiques rendant compte de la composition corporelle et de l'anabolisme musculaire (masse maigre, force musculaire, masse grasse) par contre les caractéristiques comportementales et en particulier sexuelles semblent procèder d'un modèle à seuil : la symptomatologie est présente si la testostéronémie est inférieure à une valeur seuil, par contre, au dessus de la valeur seuil, l'effet de la testostérone n'augmentera plus de manière dose-dépendante. Les données disponibles actuellement ne permettent pas de définir de manière absolue cette valeur seuil chez le sujet âgé. Chez le sujet jeune, elle est située en dessous de la limite inférieure de la normale [2].

Dans l'état actuel des connaissances, la limite inférieure de la normale des sujets jeunes constitue la valeur seuil utilisée par la majorité des auteurs pour retenir le diagnostic de DALA et pour inclure les sujets dans les essais thérapeutiques. Une testostéronémie inférieure à la limite inférieure pour l'âge traduit un hypogonadisme sévère.

Ainsi il est important que les laboratoires donnent clairement la valeur de référence de la testostérone biodisponible (ou du rapport testostérone totale / SHBG) des hommes jeunes. Ces valeurs varient selon les méthodologies employées (dosage radioimmunologique direct ou après extraction et/ou après chromatographie).

La conduite pratique du diagnostic du déficit androgénique du sujet âgé est donnée dans la Figure 1.

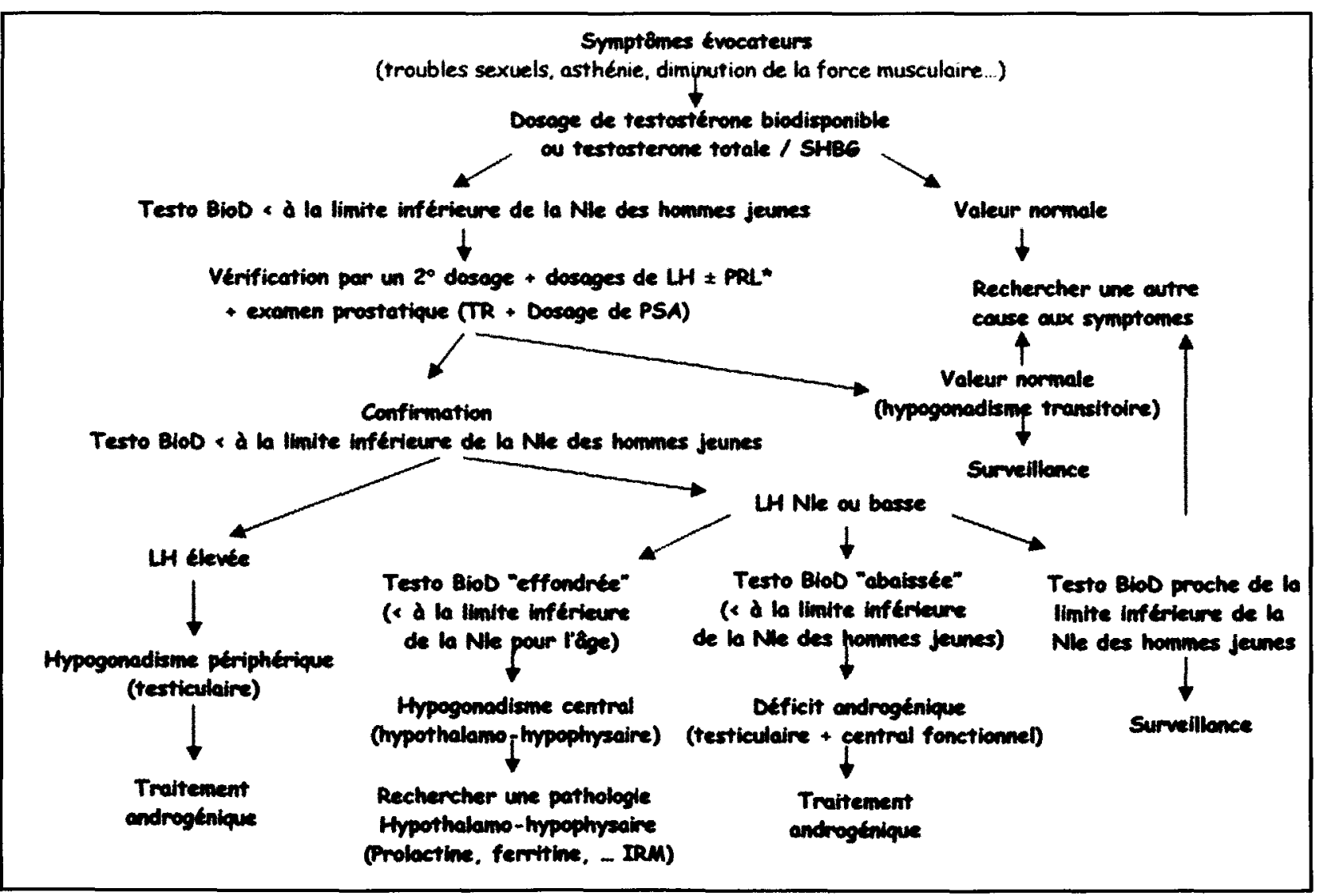

Figure 1. Diagnostic du Déficit Androgénique Lié à l'Age.

(* la mesure de la prolactine est indiquée si la LH est basse.) 
Une symptomatologie évocatrice fait réaliser un dosage de testostérone biodisponible ou à défaut un dosage de testostérone totale couplé à un dosage de SHBG. Une valeur inférieure à la limite inférieure de la normale des hommes jeunes fait suspecter un déficit androgénique. Par contre, une valeur normale fait rechercher une autre cause qu'un déficit androgénique aux symptômes.

La vérification du dosage de testostérone biodisponible couplé à un dosage de LH permet une classification physiopathologique de l'hypogonadisme et de déterminer la conduite à tenir.

- si le contrôle met en évidence une testostérone biodisponible normale, il s'agissait d'un hypogonadisme transitoire.

- si le contrôle confirme que la testostérone est inférieure à la limite inférieure de la normale des hommes jeunes, il existe un déficit androgénique.

- Une LH élevée confirme l'origine primitivement testiculaire du déficit et fait porter l'indication de traitement androgénique.

- Une LH normale ou basse donne lieu à des attitudes différentes selon l'intensité du déficit androgénique et le résultat du dosage de prolactine.

- si la testostérone biodisponible est effondrée ou si la prolactine est élevée, il faut rechercher une pathologie hypothalamo-hypophysaire par une bilan endocrinien et morphologique (IRM).

- si la testostérone biodisponible est simplement abaissée, il s'agit d'un DALA associé à une altération fonctionnelle hypothalamo-hypophysaire, ce qui fait retenir là aussi l'indication d'un traitement androgénique.

- il reste une catégorie de patients pour lesquels la testostérone biodisponible est proche de la limite inférieure de la normale des hommes jeunes et pour lesquels la LH normale ou basse ne permet pas de confirmer le déficit androgénique. Une surveillance de l'évolution sera envisagée. Pour ces cas où le diagnostic est incertain, il faut noter que le bénéfice d'un traitement androgénique, s'il existe, serait minime, en effet, les études des effets des traitements substitutifs du DALA montrent que les résultats cliniques sont d'autant plus nets que la testostéronémie était basse avant traitement $[23,24]$.

\section{POURQUOI PRESCRIRE UNE ANDROGE- NOTHERAPIE SUBSTITUTIVE ?}

Le traitement androgénique substitutif a pour objectif d'améliorer les symptômes du DALA et donc la qualité de vie. D'autres objectifs à vocation préventive (risque car- diovasculaire et ostéoporose) restent à valider par des essais cliniques prospectifs. Bien qu'il y ait encore peu d'études contrôlées de l'effet du traitement par les androgènes chez l'homme âgé, on peut tout de même dégager des conclusions préliminaires des travaux rapportant les effets de l'androgénothérapie chez le sujet âgé présentant un déficit androgénique. On trouvera dans la revue récente de la littérature de Matsumoto [14] une analyse détaillée des essais thérapeutiques de la testostérone réalisés spécifiquement chez des sujets âgés. Un résumé en est donné dans le Tableau 2

\section{Améliorer certains troubles sexuels, fonctionnels et neuropsychiques}

Le traitement androgénique chez l'homme âgé améliore la libido, l'activité sexuelle et la sensation de bien être dans un certain nombre d'études contrôlées. Le désir sexuel, les érections spontanées (nocturnes ou matinales) sont manifestement dépendantes de la testostérone. Mais chez le sujet âgé, le traitement androgénique substitutif n'améliore à lui seul qu'environ $50 \%$ des problèmes d'érection associés au DALA, car il existe souvent des facteurs vasculaires associés ; il améliore un peu plus souvent les problèmes de libido.

Il existe également une amélioration de la qualité du sommeil, une diminution de la fatigue.

La présence de récepteurs de la testostérone dảns les aires cérébrales impliquées dans la mémoire temporo-spatiale peut expliquer l'amélioration des fonctions cognitives rapportées dans plusieurs essais d'androgénothérapie. Ces effets ont été démontrés chez les sujets hypogonadiques jeunes. Les données manquent encore pour l'affirmer dans le cadre du DALA, sauf en ce qui concerne les performances spatiales.

\section{Améliorer le comportement et la qualité de vie}

Globalement, dans certaines études, l'androgénothérapie améliore l'humeur, les comportements et la sensation de bien-être s'il s'agit d'une substitution justifiée et adéquate. A l'inverse, administrer de la testostérone en excès pourrait favoriser l'agressivité.

Cela amène les experts à plaider pour une substitution adaptée et raisonnable, la plus physiologique possible, ne devant être entreprise que si l'on observe la corrélation des anomalies cliniques et biologiques.

\section{Une action très favorable sur la composition cor- porelle et la force musculaire}

Une dizaine d'études réalisées depuis 1992 ont évalué les effets de l'androgénothérapie substitutive sur la composition corporelle chez des sujets de plus de 50 ans. Même si les critères à l'inclusion sont très hétérogènes (âge, seuil de 
Tableau 2 : Effets du traitement par la Testostérone chez les hommes âgés : résultats des études contrôlées. D'après Matsumoto [13].

Effets bénéfiques

Composition corporelle, muscle

$$
\begin{aligned}
& +: \uparrow \text { Masse maigre } \\
& +: \downarrow \text { Masse grasse } \\
& \pm: \uparrow \text { Force musculaire }
\end{aligned}
$$

OS

$$
\begin{aligned}
& +: \uparrow \text { Densité osseuse } \\
& ?: \downarrow \text { Fractures }
\end{aligned}
$$

Sexualité, Bien-être

$$
\begin{aligned}
& +: \uparrow \text { Libido } \\
& 0: \text { Dysfonction érectile } \\
& +: \uparrow \text { Bien-être } \\
& ?: \downarrow \text { Dépression }
\end{aligned}
$$

\section{Fonction Cognitives}

$$
+: \uparrow \text { Orientation spaciale }
$$

$+: \uparrow$ Mémoire verbale

0 : Mémoire visuelle

$?: \downarrow$ Démences

Lipides

$+: \downarrow$ Cholesterol total et LDL

Vaisseaux

$+: \downarrow$ Angor et ischemie d'effort

$?: \downarrow$ Evènements cardiovasculaires

Etat général, état de validité

$?: \uparrow$ Forme physique

$?: \downarrow$ Fragilité générale (Frailty)

? : $\uparrow$ Qualité de vie

\section{Risques}

Erythropoïèse

$+: \uparrow$ Hématocrite,

$+: \uparrow$ Risque de polyglobulie

Apnées du sommeil

0 : Apnées du sommeil

Prostate

0 : Symptômes urinaires

0 : Taille de la prostate

0 : Débimétrie urinaire

$\pm: \uparrow$ PSA

? : $\uparrow$ Intervention pour HBP

$?: \uparrow$ Cancer de la prostate

Risques cardiovasculaires

0 : Cholestérol HDL

$?: \uparrow$ Evènements cardiovasculaires

+ : la plupart des études trouvent un effet significatif sur l'effet indiqué ;

\pm : certaines études trouvent un effet significatif sur l'effet indiqué, d'autres études n'en trouvent pas ;

0 : la plupart des études ne trouvent pas de modification sur l'effet indiqué ;

? : les études disponibles n'ont pas assez de puissance pour permettre des conclusions sur l'effet indiqué ;

$\uparrow:$ augmentation.

$\downarrow$ : diminution. 
testostéronémie initiale), on peut en tirer des conclusions claires.

La restauration d'un taux physiologique d'androgènes :

- accroît la masse maigre et plus spécifiquement la masse musculaire,

- réduit la masse grasse ;

- une augmentation de la force musculaire tant au niveau des membres supérieurs qu'inférieurs est moins constamment mise en évidence.

Même si la majorité de ces études incluent peu d'hommes âgés de plus de 65 ans, l'étude prospective randomisée de Snyder [24], chez une centaine d'hommes âgés de plus de 65 ans suivis pendant 3 ans, étaye bien le bénéfice que peut apporter une supplémentation androgénique sur la composition corporelle chez ces hommes.

\section{Un effet protecteur coronarien ?}

L'histoire des relations entre androgènes et maladie coronaire est ancienne et riche en rebondissements : entre 1930 et 1940 , un effet aussi bien préventif que curatif sur la maladie coronaire a été évoqué - il est vrai à partir de données relativement empiriques. Au contraire entre les années 50 et 80 , on a redouté des effets délétères des androgènes à partir de modèles expérimentaux peu pertinents et de situations cliniques très particulières, non représentatives.

Les données obtenues au cours des dix dernières années ne confirment pas ces craintes et vont même dans le sens d'un effet coronaro-protecteur des androgènes naturels administrés à l'homme :

- dans diverses espèces animales et sur plusieurs modèles d'athérogenèse, on retrouve un effet neutre, voire protecteur des androgènes sur le développement de lésions athéromateuses,

- les études épidémiologiques longitudinales ne retrouvent pas de corrélation, ni positive, ni négative, entre les taux sanguins d'hormones mâles et la survenue ultérieure d'événements coronariens (chez des sujets initialement indemnes de maladie athéromateuse),

- les coronariens ont le plus souvent des taux sanguins d'androgènes inférieurs à ceux de sujets témoins,

- des taux bas de testostérone totale et biodisponible ont été rattachés à un risque accru d'athérome aortique dans une vaste population masculine $(\mathrm{N}>650)$ [7]. Par ailleurs, une réduction du taux de testostérone s'associe à une augmentation de la fréquence du diabète de type II, dont on connaît les liens étroits avec les facteurs de risques cardio-vasculaires [18].

- surtout, des essais contrôlés contre placebo ont montré que l'administration de testostérone à dose unique [20] ou pendant 15 jours [5] entraînait une amélioration des performances d'effort chez des coronariens avérés : cet effet anti-ischémique est probablement la conséquence d'une vasodilatation coronaire directe.

Ces données évoquant un effet bénéfique des androgènes sont cependant préliminaires : seule une analyse prospective, idéalement contrôlée ou au moins un suivi de cohorte de vastes populations permettra de confirmer ces données préliminaires encourageantes.

La diminution du cholestérol totale et LDL observée lors du traitement substitutif chez les sujets hypogonadiques jeunes se retrouve chez les sujets âgés, alors que la baisse du cholestérol HDL mise en évidence chez les sujets jeunes ne se retrouve pas chez les sujets plus âgés [14].

\section{Une prévention de l'ostéoporose ?}

On sait depuis longtemps que l'hypogonadisme est une étiologie possible de l'ostéoporose chez l'homme : la fréquence varie selon les séries de 5 à $33 \%$ en raison de biais de recrutement et de variations dans la définition même de l'hypogonadisme.

De nombreux arguments laissent penser que l'action des androgènes sur le tissu osseux est double :

- directe par le rôle stimulant de la testostérone sur la formation osseuse

- indirecte par l'intermédiaire de l'estradiol (produit par conversion de la testostérone, sous l'influence de l'aromatase). Cette action indirecte pourrait être la plus importante comme le suggèrent les résultats de la "MINOS study " établissant une corrélation négative entre œstradiolémie biodisponible et risque ostéoporotique chez l'homme [25].

Quoi qu'il en soit, plusieurs études ont mis en évidence les effets de la testostérone sur l'os. Les études à court terme objectivent une diminution des marqueurs de la résorption osseuse, les variations des marqueurs de l'ostéoformation étant plus difficiles à interpréter.

Surtout, les essais comportant une administration prolongée de testostérone, à doses suffisantes, mettent en évidence une augmentation de la densité osseuse au niveau du rachis lombaire. En particulier l'étude d'Anderson [1], ouverte, sans groupe contrôle, chez 21 patients ostéoporotiques mais eugonadiques, montre que : l'administration de $250 \mathrm{mg}$ d'esters de testostérone (en I.M.) tous les 15 jours entraîne un gain de densité osseuse lombaire de $5 \%$, au bout de six mois ; on note parallèlement une réduction significative des marqueurs biochimiques de la résorption osseuse.

En conclusion, la supplémentation hormonale par la testostérone est efficace et indispensable devant une ostéoporose 
associée à un hypogonadisme franc. L'intérêt de la même thérapeutique dans l'ostéoporose primitive du sujet âgé demande encore à être validé par des études complémentaires.

\section{LES CONTRE-INDICATIONS ET PRÉ- CAUTIONS D'EMPLOI}

Le cancer de la prostate, supposé ou confirmé, est une contre-indication formelle à l'initiation et/ou à la poursuite d'un traitement androgénique. Bien qu'il soit établi que les androgènes contribuent directement à la promotion de la croissance prostatique, il n'est cependant pas démontré qu'ils soient à même de favoriser le développement de nouveaux cancers de la prostate. Par ailleurs, il n'existe pas de corrélation entre concentration des stéroïdes sexuels et développement des affections malignes de la prostate (pour références voir [6]).

L'hypertrophie bénigne de la prostate (HBP) ne représente pas une contre-indication à la supplémentation androgénique. Les données disponibles montrent une augmentation de volume de la prostate en début de traitement puis une stabilisation et une évolution parallèle à celle de la population générale. Une étude récente rapporte l'apparition de troubles mictionnels chez environ $12 \%$ de sujets d'une population d'hommes de plus de 50 ans traitées jusqu'à 42 mois [26], ce qui reste comparable aux données rapportées dans la population générale [21]. En cas de troubles mictionnels préexistants, le traitement androgénique sera retardé jusqu'à normalisation de l'équilibre mictionnel [16]. Toutefois, la plupart des études ayant exclu les patients porteurs d'HBP, une étude spécifique portant sur de tels patients serait utile (pour références voir [4]).

En pratique, un examen prostatique rigoureux (TR, PSA) est nécessaire avant l'instauration du traitement et au cours du traitement : dès 3 mois après le début du traitement puis semestriel et annuel par la suite ; ce suivi devra se prolonger aussi longtemps que le traitement.

La réalisation d'éventuelles biopsies prostatiques pourra être décidée, selon les règles habituelles : une biopsie préthérapeutique ne s'impose pas si TR et PSA sont normaux. L'échographie n'est pas utile pour dépister le cancer de la prostate.

Au moindre doute, un avis urologique est indispensable.

- Le cancer du sein est une contre-indication classique et absolue à l'utilisation des androgènes.

- Les apnées du sommeil représentent une précaution d'emploi, les androgènes pouvant entraîner des exacerbations nécessitant parfois un traitement par pression positive. Le risque est particulièrement élevé chez les obèses et chez les sujets présentant une insuffisance respiratoire chronique.
En stimulant l'hématopoïèse, les androgènes peuvent favoriser une polyglobulie pouvant favoriser des accidents thrombotiques, plus particulièrement chez les obèses, les sujets présentant une insuffisance respiratoire chronique et chez les fumeurs. L'hématocrite doit être contrôlé sous traitement tous les 6 mois pendant 2 ans, puis tous les ans.

Il n'y a pas de contre-indication cardiologique à la prescription d'androgènes en dehors de l'insuffisance cardiaque décompensée en raison du risque oedémateux.

En cas d'insuffisance hépatique sévère, le traitement par androgènes pourrait également exposer au risque oedémateux.

- Chez l'homme adulte, il n'existe pas de contre-indication liée à l'âge.

Une hyperprolactinémie doit être écartée, du fait du risque d'induire une poussée évolutive d'une éventuelle tumeur par l'aromatisation des androgènes en œstrogènes.

\section{COMMENT PRESCRIRE LA TESTOSTÉRONE ?}

L'objectif du traitement est de restaurer l'état fonctionnel, physique et comportemental du patient, en ramenant la testostéronémie au plus proche de la physiologie.

\section{Quelles molécules, quelles modalités d'admi- nistration?}

Les différentes formes d'androgénothérapie substitutive utilisées font appel soit à la testostérone base, soit à des esters d'acides gras : énanthate injectable ou undécanoate administrable per os. L'androstanolone (dihydrotestostérone) a une place à part.

Au choix du mode d'administration de la testostérone ou de ses esters sont attachés des avantages et inconvénients très différents pour chaque formulation.

Les injections intramusculaires d'ester de testostérone (énanthate de testostérone), parfois douloureuses du fait de l'excipient huileux, ne nécessitent qu'une injection de 250 mg en moyenne toutes les 3 semaines. Leur inconvénient principal est d'ordre pharmacocinétique, avec une grande fluctuation des taux de testostérone. Le pic est précoce et supraphysiologique, avec un retour progressif dans la zone de l'hypoandrogénie. Il peut en résulter sur le plan clinique une sensibilité mammaire ou le développement d'une gynécomastie du fait des taux supraphysiologiques survenant après les injections. Les patients décrivent souvent des fluctuations de l'humeur, du comportement, de la libido et de la fonction sexuelle. Une meilleure stabilité de la testostéronémie peut être obtenue par l'injection plus fréquente de doses moindres.

Le traitement per os permet une adaptation progressive 
des doses. En France, les formes méthylées, susceptibles d'engendrer une hépatotoxicité, ne sont plus commercialisées. Par contre, l'undécanoate de testostérone, gagne la circulation générale par les lymphatiques intestinaux, ce qui permet d'éviter le passage hépatique. L'absorption de l'undécanoate de testostérone, 120 à $160 \mathrm{mg}$ en 2 à 3 prises par jour, doit obligatoirement avoir lieu lors d'un repas pour que l'absorption lymphatique soit effective. La cinétique de l'absorption intestinale et du passage par voie lymphatique donne lieu à des fluctuations de la testostérone plasmatique au cours de la journée ce qui rend difficile l'utilisation des dosages de testostérone pour adapter la posologie.

La voie percutanée et transdermique est utilisée dans certains pays sous forme de patchs de testostérone à appliquer sur le scrotum ou en dehors du scrotum, et depuis peu en France, sous forme de gel. Elle permet un monitoring pharmacologique.

Les patchs, scrotaux ou non scrotaux d'application quotidienne (non disponibles en France à ce jour) ont pour principal inconvénient leur galénique : leur surface importante (environ $60 \mathrm{~cm}^{2}$ ) entraîne de fréquentes réactions cutanées au site d'application. Les patchs d'application quotidienne sur le scrotum nécessitent un rasage fréquent de la peau du scrotum, et leur application est plus ou moins acceptée.

Le gel de testostérone se présente sous forme d'un gel hydro-alcoolique de testostérone en sachet-dose. Il permet d'administrer la testostérone par voie percutanée. Après absorption par la peau et saturation du "réservoir cutané", la testostérone diffuse dans la circulation générale à des taux relativement constants au cours du nycthémère, donnant des taux plasmatiques de testostérone stables sur 24 heures. En une seule application, en général de 5 grammes de gel soit $50 \mathrm{mg}$ de testostérone par jour, au niveau des épaules, des avant-bras et/ou de l'abdomen, les concentrations plasmatiques de testostérone se situent à un niveau physiologique dès le 2ème jour de traitement. Cette forme d'administration de la testostérone semble avoir une bonne acceptabilité. Quelques précautions doivent être prises pour éviter la diffusion de la testostérone à l'entourage du sujet traité.

Le gel de dihydrotestostérone (androstanolone) se présente sous forme de gel hydro-alcoolique de dihydrotestostérone en tube. Il permet d'administrer par voie percutanée le métabolite androgénique le plus actif de la testostérone. À l'instar de la testostérone administrée par la même voie, la dihydrotestostérone diffuse du réservoir cutané vers la circulation générale où elle réside à des taux stables sur 24 heures. En une application quotidienne de 5 à 10 grammes de gel, soient $125 \mathrm{mg}$ à $250 \mathrm{mg}$ de dihydrotestostérone, les taux plasmatiques obtenus assurent une excellente androgénicité. Néanmoins, inductrice d'une réduction des taux plasmatiques d'œstradiol, à la fois par son action antigonadotrope et par 1'impossibilité de son aromatisation en estrogènes, la dihydrotestostérone est dépourvue des propriétés de la testostérone liée à sa transformation en œstradiol. Ceci représente une limite à l'intérêt de la substitution androgénique de l'hypogonadisme masculin par dihydrotestostérone. Ce stéroïde garde cependant tout son intérêt dans le traitement de la gynécomastie ne relevant pas directement d'une thérapeutique étiologique. Les précautions à prendre pour éviter la diffusion de la dihydrotestostérone à l'entourage du sujet traité sont les mêmes qu'avec le gel de testostérone.

\section{Quelle surveillance?}

La surveillance est essentiellement clinique : la non réversibilité des symptômes ou signes ayant motivé le traitement devant conduire à s'interroger sur la responsabilité du déficit androgénique dans la symptomatologie et sur le bien fondé de sa poursuite...

La surveillance hormonale repose sur le dosage de la testostérone selon un schéma spécifique à chaque type d'androgène. Avec les esters de testostérone injectables, la hauteur du pic peut être appréciée dans les jours suivant l'injection, l'espacement des injections peut être guidé par la concentration résiduelle de testostérone biodisponible avant l'injection suivante. Le pic de testostérone circulante survient en moyenne 5 heures après l'absorption orale d'undécanoate de testostérone, les valeurs de testostéronémie s'abaissent ensuite nécessitant une nouvelle administration au moins à la 12ème heure. Avec la forme gel, l'état d'équilibre est atteint dès le 2 ème jour de traitement et se maintient au long cours.

La surveillance prostatique rigoureuse est essentielle, reposant sur le toucher rectal et le dosage plasmatique du PSA. La première visite de contrôle est effectuée 3 mois après l'initiation du traitement et sera renouvelée tous les six mois pendant deux ans, puis tous les ans.

L'hématocrite doit être contrôlé tous les 6 mois pendant les 2 premières années, puis 1 fois par an.

\section{Quelle durée de traitement ?}

Bien qu'il n'existe pas de données consensuelles et que le recul dans les études thérapeutiques de qualité n'excède pas 4 ans, le traitement substitutif semble pouvoir être poursuivi, s'il est débuté à bon escient et dès lors que les objectifs thérapeutiques sont atteints, jusqu'à ce que survienne l'une des situations de contre-indication ou de nonindication. 
Les données actuelles de la pharmacovigilance n'ont pas fait part d'effet indésirable sévère ou inattendu.

\section{CONCLUSION}

Par rapport aux hypogonadismes du sujet jeune, le DALA se caractérise par une symptomatologie peu spécifique en raison d'une part de sa possible intrication avec d'autres manifestations liée à l'avancée en âge et d'autre part du caractère partiel du déficit androgénique. Il est encore plus important que chez le sujet jeune que le diagnostic repose sur des dosages hormonaux tenant compte de l'influence de la SHBG : testostérone biodisponible ou au moins index de testostérone libre (testostérone totale/SHBG) ou testostérone libre calculée. Après avoir éliminé un hypogonadisme transitoire et avoir écarté un hypogonadisme satellite d'une affection générale et une pathologie hypothalamo-hypophysaire susceptible de relever d'un traitement étiologique ou contre-indiquant l'androgénothérapie, la mise en place du traitement androgénique substitutif comporte aussi des particularités liées à l'âge. Les mesures de dépistage du cancer de la prostate doivent être entreprises avant tout traitement androgénique et renouvelées régulièrement au cours du traitement. II en est de même pour l'hématocrite à la recherche d'une polyglobulie. Une prise en charge globale de l'état de santé du sujet doit aussi être envisagée, il est en effet important de tenir compte des pathologies associées, en particulier des facteurs de risques cardiovasculaires venant fréquemment ajouter leurs effets propres en particulier sur les troubles de l'érection.

La surveillance de l'androgénothérapie s'attachera à trouver la posologie permettant un traitement substitutif physiologique, à surveiller l'état prostatique, l'hématocrite, à vérifier la bonne évolution des signes cliniques ayant fait découvrir le DALA et à prendre en charge les pathologies associées.

\section{REMERCIEMENTS}

Nous souhaitons remercier ici les Laboratoires BesinsInternational pour leur soutien logistique aux séances de travail et à la collecte des éléments bibliographiques.

\section{RÉFÉRENCES}

1. ANDERSON F.H., FRANCIS R.M., PEASTON R.T., WASTELL H.J. : Androgen supplementation in eugonadal men with osteoporosis: effects of six months' treatment on markers of bone formation and resorption. J. Bone Miner. Res., 1997, 12 : $472-478$

2. BAGATELL C.J., HEIMAN J.R., RIVIER J.E., BREMNER W.J. : Effects of endogenous testosterone and estradiol on sexual behavior in normal young men. J. Clin. Endocrinol. Metab., $1994,78: 711-716$.

3. BHASIN S., WOODHOUSE L., CASABURI R., et al. : Testosterone dose-response relationships in healthy young men. Am. J. Physiol. Endocrinol. Metab., 2001, 281 : E1172-1181.

4. COSTA P. : Adénome prostatique, hormones et androgénothérapie. Andrologie, 2002, 12 : 133-135.

5. ENGLISH K.M., STEEDS R.P., JONES T.H., DIVER M.J., CHANNER K.S. : Low-dose transdermal testosterone therapy improves angina threshold in men with chronic stable angina: A randomized, double-blind, placebo-controlled study. Circulation, 2000, 102 : 1906-1911

6. FLAM T. : Traitement androgénique substitutif et cancer prostatique. Andrologie, 2002, $12: 136-137$.

7. HAK A.E., WITTEMAN J.C., DE JONG F.H., GEERLINGS M.I., HOFMAN A., POLS H.A. : Low levels of endogenous androgens increase the risk of atherosclerosis in elderly men: the Rotterdam study. J. Clin. Endocrinol. Metab., 2002, 87 : 36323639.

8. HEINEMANN L.A.I., ZIMMERMANN T., VERMEULEN A., THIEL C., HUMMEL W. : A new 'aging males' symptoms' rating scale. Aging Male, 1999, 2 : 105-114.

9. KIM C.Y., BUVAT J., CARSON C.C., et al. : Endocrine and metabolic aspect including treatment. In Erectile dysfunction. 1st international consultation on erectile dysfunction, July 1-3, 1999, Paris. Jardin A., Wagner G., Khoury S., Giuliano F., PadmaNathan H., Rosen R. eds. Health Publication Ltd, Plymbridge Distributors, Plymouth, 2000 : 207-240.

10. KUHN J.M. : Déficit androgénique : effet de la substitution sur la composition corporelle. Andrologie, 2002, 12 : 17-23.

11. LEGRAND E., SIMON V., AUDRAN M. : Stéroides sexuels et ostéoporose chez l'homme. Andrologie, 2002, 12 : 24-31.

12. LEGROS J.J., DELHEZ M. : Détection de la déficience androgénique chez l'homme de plus de 50 ans : utilisation d'une version française du test ADAM. Med. Hyg., 2002, 60 : 1490-1495.

13. LEJEUNE H. : Le déficit androgénique lié à l'âge chez l'homme: terminologie, épidémiologie. Andrologie, 2002, 12 : 11-16.

14. MATSUMOTO A.M. : Andropause: clinical implications of the decline in serum testosterone levels with aging in men. J. Gerontol. A. Biol. Sci. Med. Sci., 2002, 57 : M76-99.

15. MIMOUN S. : Androgènes et qualité de vie. Andrologie, 2002, $12: 257-261$.

16. MORALES A., LUNENFELD B. : Investigation, treatment and monitoring of late-onset hypogonadism in males. Official recommendations of ISSAM. Aging Male, 2002, 5 : 74-86. 
17. MORLEY J.E., CHARLTON E., PATRICK P., et al. : Validation of a screening questionnaire for androgen deficiency in aging males. Metabolism, 2000, $49: 1239-1242$.

18. OH J.Y., BARRETT-CONNOR E., WEDICK N.M., WINGARD D.L. : Endogenous sex hormones and the development of type 2 diabetes in older men and women: the Rancho Bernardo study. Diabetes Care, 2002, 25 : 55-60.

19. ROGER M., LAHLOU N. : Le déficit androgénique lié à l'âge : du diagnostic biologique au traitement substitutif. Andrologie, 2002, $12: 138-146$.

20. ROSANO G.M., LEONARDO F., PAGNOTTA P., et al. : Acute anti-ischemic effect of testosterone in men with coronary artery disease. Circulation, 1999, 99 : 1666-1670.

21. SAGNIER P.P., MACFARLANE G., RICHARD F., BOTTO H., TEILLAC P., BOYLE P. : Results of an epidemiological survey using a modified American Urological Association symptom index for benign prostatic hyperplasia in France. J. Urol., 1994, $151: 1266-1270$.

22. SMITH K.W., FELDMAN H.A., MCKINLAY J.B. : Construction and field validation of a self-administered screener for testosterone deficiency (hypogonadism) in ageing men. Clin. Endocrinol. (Oxf), 2000, $53: 703-711$.

23. SNYDER P.J., PEACHEY H., HANNOUSH P., et al. : Effect of testosterone treatment on bone mineral density in men over 65 years of age. J. Clin. Endocrinol. Metab., 1999, 84 : 1966-1972.

24. SNYDER P.J., PEACHEY H., HANNOUSH P., et al. : Effect of testosterone treatment on body composition and muscle strength in men over 65 years of age. J. Clin. Endocrinol. Metab., 1999, 84 : 2647-2653.

25. SZULC P., MUNOZ F., CLAUSTRAT B., et al. : Bioavailable estradiol may be an important determinant of osteoporosis in men: the MINOS study. J. Clin. Endocrinol. Metab., 2001, 86 : 192-199.

26. WANG C., SWEDLOFF R.S., IRANMANESH A., et al. : Long term efficacy and safety of transdermal testosterone gel (Androgel) in hypogonadal men. The Endocrine Society's 84th Annual Meeting, 2002, Abstract : P2-646.

\section{ABSTRACT}

\section{MULTIDISCIPLINARY AGREEMENT ON DIAGNOSIS AND TREATMENT OF ANDROGENIC DEFICIENCY IN AGING MALE}

\author{
Multidisciplinary group : Jacques BUVAT, Pierre \\ COSTA, Thierry FLAM, François GIULIANO, \\ Jean-Marc KUHN, Erick LEGRAND, Hervé LEJEUNE, \\ Sylvain MIMOUN, Marc ROGER, Simon WEBER.
}

The diagnosis of the androgen deficiency of the aging male (ADAM) is suspected in the presence of relatively unspecific clinical symptoms. The biological evidence of androgen deficiency should be given by using an assay taking into account the level of the sex hormone binding protein (SHBG), such as the bioavailable testosterone assay or, at least, the free testosterone index or the calculated free testosterone which both require measuring total testosterone and SHBG levels. Although the threshold value for defining ADAM has not been fully investigated, the lower limit of normal values in healthy young men which is commonly used for including subjects in therapeutic trials, seems appropriate. According to the currently available data, testosterone replacement therapy in hypogonadal aging men seems to be beneficial to quality of life, sexuality, metabolic status, body composition and osteoporosis. The initiation of androgen replacement therapy requires a careful screening for prostate cancer. Prostate and hematocrit must be monitored during the replacement therapy which is intended for maintaining testosterone levels in the physiological range. Associated disease should be accounted for as a possible factor worsening ADAM and could be relevant of a specific therapy.

Key Words : Andropause, androgenic deficiency, aging, testosterone 